\section{RMD Open}

Rheumatic \&

Musculoskeletal Diseases

\title{
Comparative effectiveness of biologics and targeted therapies for psoriatic arthritis
}

Hanzhe Zhang, ${ }^{1}$ Jiajun Wen, ${ }^{1,2}$ G Caleb Alexander (1) , ${ }^{1,3,4}$ Jeffrey R Curtis (1) ${ }^{5,6}$

To cite: Zhang H, Wen J, Alexander GC, et al. Comparative effectiveness of biologics and targeted therapies for psoriatic arthritis. RMD Open 2021;7:e001399. doi:10.1136/ rmdopen-2020-001399

- Additional material is published online only. To view, please visit the journal online (http://dx.doi.org/10.1136/ rmdopen-2020-001399).

Received 16 July 2020 Revised 30 December 2020 Accepted 4 January 2021

\section{Check for updates}

C) Author(s) (or their employer(s)) 2021. Re-use permitted under CC BY-NC. No commercial re-use. See rights and permissions. Published by BMJ.

For numbered affiliations see end of article.

Correspondence to Dr Jeffrey R Curtis; jcurtis@uab.edu, jrcurtis@ uabmc.edu

\section{ABSTRACT}

Objective To quantify comparative effectiveness of interleukin (IL)-12/23 antagonist (ustekinumab), IL17A antagonists (secukinumab and ixekizumab), PDE4 inhibitor (apremilast) and tumour necrosis factor-alpha (TNF- $\alpha$ ) inhibitors (infliximab, etanercept, adalimumab, certolizumab pegol and golimumab) for psoriatic arthritis (PsA).

Methods We adapted a deidentified claims-based algorithm validated for inflammatory arthritis treatments to compare treatments among a retrospective cohort of commercially insured and Medicare Advantage beneficiaries with PsA from October 2013 to April 2019 in the OptumLabs Data Warehouse. Main outcomes include (1) treatment effectiveness, based on: adherence, adding or switching biologic or PDE4, addition of new non-biologic disease-modifying antirheumatic drug, increase in biologic or PDE4 dose or frequency and glucocorticoid use and (2) percentage of each group fulfilling the effectiveness algorithm. We used Poisson regression with robust variance stratified by prior PsA biologic exposure and adjusted for potential confounders.

Results Of 2730 individuals with PsA, 327 received IL-12/23, 138 IL-17A's, 624 PDE4 and 1641 TNF- $\alpha$ 's. Effectiveness criteria were fulfilled among $63(19.3 \%)$ IL-12/23 recipients, 40 (29.0\%) IL-17A recipients, 160 (25.6\%) PDE4 recipients and 530 (32.3\%) TNF- $\alpha$ recipients. Among biologic-naïve individuals, IL-12/23 was less effective than TNF- $\alpha$ 's with fully adjusted relative risk (aRR) compared with TNF- $\alpha$ 's of $0.63(95 \% \mathrm{Cl} 0.45$ to 0.89 ). Among biologic-experienced individuals, PDE4 recipients were less effective than TNF- $\alpha$ 's (aRR 0.67, $95 \% \mathrm{Cl} 0.46$ to 0.96 ).

Conclusions TNF- $\alpha$ 's appeared more effective than IL-12/23's for biologic-naïve individuals, and PDE4's for biologic-experienced individuals. These results may help inform treatment choice for individuals with PsA.

\section{INTRODUCTION}

Ustekinumab (an interleukin (IL) 12-23 antagonist), secukinumab and ixekizumab (both IL-17A antagonists) are recently introduced biologics approved by the European Medicines Agency ${ }^{1-3}$ and US Food and Drug Administration for treatment of psoriatic arthritis (PsA) ${ }^{4-6}$ In the absence of multiple

\section{Key messages}

What is already known about this subject?

- Interleukin (IL)-12/23 antagonist, IL-17A antagonists, PDE4 inhibitor and tumour necrosis factoralpha (TNF- $\alpha$ ) inhibitors are among available biologics and treatments for psoriatic arthritis (PSA).

- Previous studies suggest TNF- $\alpha$ and IL-17A inhibitors generally performed better than IL-12/23 and PDE4.

What does this study add?

- TNF- $\alpha$ 's appeared more effective than IL-12/23's among biologic-naïve individuals, and more effective than PDE4's among biologic-experienced individuals.

How might this impact on clinical practice?

- Findings may help inform treatment options for individuals with PsA.

large-scale head-to-head randomised clinical trials comparing PsA therapies to one another, systematic reviews and meta-analyses have compiled randomised controlled trials of these products to compare their efficacy with that of tumour necrosis factor-alpha (TNF- $\alpha$ ) inhibitors. ${ }^{78}$ One network meta-analysis found that when comparing American College of Rheumatology (ACR) response rates, adalimumab and secukinumab performed better than apremilast and ustekinumab, while golimumab and infliximab performed better than most treatments aside from adalimumab and secukinumab among biologic-naïve individuals. ${ }^{7}$ Another meta-analysis showed secukinumab $300 \mathrm{mg}$ performed better than apremilast on ACR20 in a general population, while among the TNF- $\alpha$ naïve population secukinumab performed better over apremilast and ustekinumab. ${ }^{8}$ Despite the insights these studies provide towards TNF- $\alpha$ and IL-17A inhibitors generally performing better than IL-12/23 and PDE4, their generalisability is limited because they are based 
on randomised trials rather than real-world use, and thus reflect restrictive inclusion criteria, close participant monitoring and short durations of assessments. ${ }^{7-9}$

Real-world studies using administrative claims data can complement insights from clinical trials as well as support assessments of value-based care, but such data often miss clinical covariates and outcomes of interest, including measures of treatment effectiveness. ${ }^{9}$ To address the limitations of clinical trials and to inform the utility of a claims-based approach for evaluating treatment effectiveness in PsA using administrative data, we adapted a previously validated algorithm that characterised effectiveness of treatments for rheumatoid arthritis (RA). ${ }^{10}$ In this study, we assessed the comparative effectiveness of IL-12/23, IL-17A, PDE4 and TNF- $\alpha$ for PsA.

\section{METHODS}

\section{Data source and study design}

This retrospective cohort study used deidentified claims data with linked socioeconomic status information, including commercially insured patients contained within the OptumLabs Data Warehouse. ${ }^{11}$ The data warehouse has deidentified administrative claims and electronic health record data of ambulatory and inpatient care on over 200 million enrollees with varied demographic makeup which encompasses those living in the USA with commercial or Medicare Advantage coverage. ${ }^{11} 12$

\section{Patient and public involvement}

No patients were involved in design, conduct or writing of this study.

\section{Study cohort}

We used a validated, claims-based algorithm to identify individuals with PsA, requiring each individual to have two or more diagnostic codes for PsA from a rheumatologist (International Classification of Diseases, Ninth Revision, Clinical Modification [ICD-9-CM] 696.0; ICD-10-CM L40.50, L40.51, L40.52, L40.53, L40.54 and L40.59) or at least one of these codes from a rheumatologist combined with one or more diagnosis code of psoriasis from a dermatologist (ICD-9-CM 696.1; ICD-10-CM L40.X except L40.5). ${ }^{13} 14$ The positive predictive value of this algorithm to accurately identify patients with PsA was $80 \% .{ }^{13}$ We included individuals with commercial or Medicare Advantage coverage and required them to be continuously enrolled with medical and pharmacy coverage for at least 6 months before and 12 months after the index date. Index date was defined as the date of the first claim for a relevant exposure of interest. We excluded any individual based on all available data from prior to index date through the follow-up period with any of the following indications including Crohn's disease, ankylosing spondylitis, RA, juvenile idiopathic arthritis, ulcerative colitis or hidradenitis suppurativa. Exclusions for ankylosing spondylitis or RA also required at least one diagnosis code by a rheumatologist. Patients had to have been 18 years or older on the date of their first treatment claim or procedure between 1 October 2013 and 1 April 2018.

\section{Exposures of interest}

The treatment from each patient's earliest start date that falls within the period was used as their starting treatment group, with each patient allowed just one treatment period for cohort entry. Patients must have one or more medical or prescription claim for IL-12/23 (ustekinumab), IL-17A (secukinumab and ixekizumab), PDE4 (apremilast) or one of the following TNF-alpha inhibitors (infliximab, etanercept, adalimumab, certolizumab pegol or golimumab). Biosimilars were grouped according to their bio-originator reference exposure. For individuals in the TNF group, a TNF-experienced subgroup was created by searching for the next switch date for those who first initiated TNF within the study period. These individuals were followed to look for their next switch to a different TNF before the end of the study period, and if any switch existed, the switch date along with the TNF they switched to became their only assigned biologic.

Patients could not have had claims for more than one biologic on the index date, or have had both pharmacy and procedure claims for the same treatment of interest if they were in an exposure group for that treatment due to conversion and handling for certain criteria for effectiveness analysis. ${ }^{15}$

\section{Algorithm}

We used a claims-based algorithm to define treatment effectiveness. The adapted algorithm (online supplemental eTable 1) used claims data to proxy for effective treatment for RA, based on observable patterns of care for what rheumatologists and patients do in response to a PsA therapy that was not working. ${ }^{10}$ The algorithm was validated against the gold standard of either low disease activity or significant improvement in disease activity measured using the Disease Activity Score in 28 joints, a commonly used measure in PsA and RA clinical trials. It has been shown to have sensitivity, specificity, positive and negative predictive values all ranging from $75 \%$ to $90 \% .{ }^{10}$ In this algorithm, all six criteria must be met for the treatment to be deemed effective, and assessment is commonly made at 12 months after start of therapy. ${ }^{10}$ The criteria for which the treatment would be considered effective includes high adherence (medication possession ratio of $\geq 80 \%$ ), no switching to another PsA therapy, lack of dose escalation of the initial PsA therapy, no addition of a conventional synthetic immunomodulatory drug for PsA (eg, methotrexate, cyclosporine), no increased use of systemic glucocorticoids compared with baseline, and no more than a single intraarticular joint injection after 90 days. ${ }^{10}$

\section{Covariates}

We derived information from the claims data including individuals' age at index date, gender, race or ethnicity, 
educational level, income and Deyo Charlson Comorbidity Index score. ${ }^{14} 16$ We also assessed information regarding use of treatments 6 months prior to index date including phototherapy, non-steroidal anti-inflammatory drugs (NSAIDs), disease-modifying antirheumatic drugs (DMARDs), oral glucocorticoid use, opioid claims, as well as a count for the number of previous biologics including abatacept, brodalumab, guselkumab and tildrakizumab. ${ }^{14}$ We assessed healthcare utilisation with inpatient and emergency department stays. We also assessed the presence of chronic obstructive pulmonary disease, asthma, diabetes, fibromyalgia, any mood disorders and major depressive disorder (MDD) during any time before the index date. Covariates were selected based on their potential role as confounders of main exposureoutcome associations using subject matter expertise.

\section{Analysis}

We defined our primary outcome as treatment effectiveness represented by meeting all six criteria depicted in online supplemental eTable 1 . In secondary analyses, we assessed the fulfilment of individual criteria. For overall effectiveness, we assessed pairwise comparisons with TNF- $\alpha$ using $\chi^{2}$ test. We examined the bivariate association between each exposure of interest and our primary outcome and built multivariate models using Poisson regression with robust variance. ${ }^{17}$ To do so, we included basic demographic characteristics (eg, age, sex) and variables of at least borderline statistical significance with the outcome in bivariate analysis $(p<0.10)$. Model fit was assessed through goodness-of-fit $\chi^{2}$ test. Results from both the model including age, sex and race and a separate full adjusted model including significant variables were included.

\section{Sensitivity analyses}

In sensitivity analyses, we stratified individuals into those who were biologic-naïve versus biologic-experienced and performed individual subgroup analysis within each stratum. This stratification was decided on a priori, based on the possibility that biologic-naive patients might have a differential treatment response to certain therapies compared with biologic-experienced patients. Because this stratification was preplanned based on subject matter expertise, no formal interaction terms were tested. We defined biologic-naïve individuals as those with no counts of previous psoriasis or PsA biologic at any time prior to the index date for any primary treatment of interest. We also tested a modified outcome which no longer required that individuals not increase their biologic or PDE4 dose or frequency during the effectiveness assessment period, dropping the fourth criteria for effectiveness.

\section{RESULTS}

\section{Characteristics of cohort}

In total, 2730 patients were included (table 1 ). There were 327 patients in IL-12/23 group, 138 in IL-17A group, 624 in PDE4 group and 1641 in TNF- $\alpha$ group. The different exposure groups were similar with respect to age, sex and race or ethnicity. However, there were differences across groups. For example, compared with TNF- $\alpha$ group (46.1\%), lower proportions of IL-12/23 (31.8\%), IL-17A $(26.8 \%)$ and PDE4 $(32.1 \%)$ groups had DMARDs use 6 months prior to index date. A greater proportion of individuals in the IL-17A group $(25.4 \%)$ had MDD compared with all other exposure groups $(15.6 \%-18.9 \%)$.

\section{Crude effectiveness across treatment groups}

Table 2 depicts the proportion of each exposure group fulfilling different effectiveness criteria. Overall, 63 $(19.3 \%)$ in the IL-12/23 group met all six effectiveness criteria along with 160 (25.6\%) in PDE4 group, 40 $(29.0 \%)$ in the IL-17A group and $530(32.3 \%)$ in TNF- $\alpha$ group. High treatment adherence had the lowest fulfilment rates across all exposure groups, ranging from $26.0 \%$ to $42.0 \%$.

\section{Adjusted effectiveness across treatment groups}

Table 3 describes partially and fully adjusted comparative effectiveness of the exposures examined. After adjustment for individuals' age, sex and race or ethnicity, partially adjusted relative risk (aRR) for effectiveness compared with TNF- $\alpha$ 's was lower for IL-12/23 and for PDE4 therapy, but not significantly different for IL-17A. In the fully adjusted model, the aRR for effectiveness compared with TNF- $\alpha$ 's was lower for IL-12/23's (aRR $0.75,95 \%$ CI 0.59 to 0.95 ), and not significantly different for IL-17A's (aRR 1.30, 95\% CI 0.99 to 1.71 ) nor for PDE4's (aRR $0.90,95 \%$ CI 0.77 to 1.04 ).

Table 3 also depicts the same analyses without requiring the absence of treatment dose or frequency escalation during the observation period. Results were similar except for fully adjusted results, which had IL-17A with a higher aRR for effectiveness compared with TNF (aRR $1.50,95 \%$ CI 1.18 to 1.91 ) while PDE4 had a lower aRR for effectiveness (aRR $0.85,95 \%$ CI 0.73 to 0.99 ).

\section{Adjusted effectiveness stratified by prior biologic exposure}

Table 4 shows the comparisons after multivariable adjustment stratified by biologic-naïve versus biologicexperienced subgroups. Among the 1774 patients in the biologic-naïve cohort, after adjusting for individuals' age, sex and race, IL-12/23 was less effective than TNF- $\alpha$ 's while there was no statistically significant difference between IL-17A or PDE4 effectiveness compared with TNF- $\alpha$ 's. In the same cohort, after full adjustment and compared with TNF- $\alpha$ 's, the aRR for IL-12/23 was significantly lower (aRR 0.63 , 95\% CI 0.45 to 0.89 ), but not significantly different for IL-17A's (aRR 1.02, 95\% CI 0.65 to 1.58 ) nor for PDE4 (aRR 0.92, 95\% CI 0.78 to 1.09 ).

For the 956 patients in the biologic-experienced cohort, the sex, age and race adjusted $R R$ for effectiveness compared with TNF- $\alpha$ 's was not significantly different for IL-12/23 nor IL-17A's, but was lower for PDE4. After full adjustment, there was no difference in the comparative effectiveness of IL-12/23 (aRR 0.77, 95\% CI 0.55 to 1.09) 
Table 1 Baseline characteristics of individuals with psoriatic arthritis grouped by treatments of interest $(N=2730)$

\begin{tabular}{|c|c|c|c|c|}
\hline Characteristics & IL-12/23 (N=327) & $\mathrm{IL-17A}^{*}(\mathrm{~N}=138)$ & PDE4 (N=624) & TNF-alpha† ( $\mathrm{N}=1641)$ \\
\hline Age, mean (SD) & $52.3(11.6)$ & $52.1(10.4)$ & $52.8(12.0)$ & $49.6(12.7)$ \\
\hline Female, N (\%) & $180(55.0)$ & $72(52.2)$ & $335(53.7)$ & 867 (52.8) \\
\hline \multicolumn{5}{|l|}{ Race/ethnicity, N (\%) } \\
\hline White & $240(73.4)$ & $88(63.8)$ & $443(71.0)$ & $1123(68.4)$ \\
\hline Asian & $11(3.4)$ & $\ddagger$ & $14(2.2)$ & $51(3.1)$ \\
\hline Black & $20(6.1)$ & $\ddagger$ & $37(5.9)$ & $92(5.6)$ \\
\hline Hispanic & $25(7.6)$ & $\ddagger$ & $41(6.6)$ & $126(7.7)$ \\
\hline Unknown & $13(4.0)$ & $11(8.0)$ & $39(6.2)$ & $52(3.2)$ \\
\hline Missing & $18(5.5)$ & $20(14.5)$ & $50(8.0)$ & $197(12.0)$ \\
\hline \multicolumn{5}{|l|}{ Education, N (\%) } \\
\hline$<12$ th grade & $\ddagger$ & $\ddagger$ & $\ddagger$ & $\ddagger$ \\
\hline High school diploma & $72(22.0)$ & $26(18.8)$ & $121(19.4)$ & $353(21.5)$ \\
\hline Less than bachelor & $169(51.7)$ & $70(50.7)$ & 330 (52.9) & 803 (48.9) \\
\hline Bachelor degree plus & $67(20.5)$ & $19(13.8)$ & $118(18.9)$ & $284(17.3)$ \\
\hline Unknown & $\ddagger$ & $\ddagger$ & $\ddagger$ & $\ddagger$ \\
\hline Missing & $\ddagger$ & $\ddagger$ & $\ddagger$ & $\ddagger$ \\
\hline \multicolumn{5}{|l|}{ Income, N (\%) } \\
\hline$<U S \$ 40000$ & $30(9.2)$ & $12(8.7)$ & $58(9.3)$ & $148(9.0)$ \\
\hline US\$40 000-US\$74 999 & $74(22.6)$ & $31(22.5)$ & $125(20.0)$ & $318(19.4)$ \\
\hline US\$75 000-US\$124 999 & $76(23.2)$ & $28(20.3)$ & $160(25.6)$ & $381(23.2)$ \\
\hline US\$125 000-US\$199999 & $47(14.4)$ & $20(14.5)$ & $102(16.3)$ & $223(13.6)$ \\
\hline$\geq$ US\$200 000 & $45(13.8)$ & $13(9.4)$ & $70(11.2)$ & $161(9.8)$ \\
\hline Unknown/missing & $55(16.8)$ & $34(24.6)$ & $109(17.5)$ & $410(25.0)$ \\
\hline $\begin{array}{l}\text { Deyo Charlson Comorbidity Index } \\
\text { Score, mean (SD) }\end{array}$ & $1(1.4)$ & $1.1(1.5)$ & $1.1(1.6)$ & $0.8(1.3)$ \\
\hline $\begin{array}{l}\text { Light or phototherapy, } 6 \text { months } \\
\text { preindex, } N(\%)\end{array}$ & $\ddagger$ & $\ddagger$ & $17(2.7)$ & $16(1.0)$ \\
\hline \multicolumn{5}{|l|}{ Pharmacological treatments } \\
\hline NSAIDs, 6 months preindex, $\mathrm{N}(\%)$ & $96(29.4)$ & $47(34.1)$ & $223(35.7)$ & $666(40.6)$ \\
\hline $\begin{array}{l}\text { DMARDs, } 6 \text { months preindex, } \mathrm{N} \\
\text { (\%) }\end{array}$ & $104(31.8)$ & $37(26.8)$ & $200(32.1)$ & $757(46.1)$ \\
\hline $\begin{array}{l}\text { Oral glucocorticoid, } 6 \text { months } \\
\text { preindex, } \mathrm{N}(\%)\end{array}$ & $86(26.3)$ & $47(34.1)$ & $190(30.4)$ & $581(35.4)$ \\
\hline $\begin{array}{l}\text { Opioid claims, } 6 \text { months preindex, } \\
\mathrm{N}(\%)\end{array}$ & $122(37.3)$ & 55 (39.9) & $231(37.0)$ & $541(33.0)$ \\
\hline \multicolumn{5}{|l|}{ Healthcare utilisation } \\
\hline Any inpatient stays, N (\%) & $11(3.4)$ & $\ddagger$ & $43(6.9)$ & $66(4.0)$ \\
\hline Any ED stays, N (\%) & $31(9.5)$ & $18(13.0)$ & $95(15.2)$ & $198(12.1)$ \\
\hline \multicolumn{5}{|l|}{ Comorbid conditions } \\
\hline COPD, N (\%) & $39(11.9)$ & $13(9.4)$ & $78(12.5)$ & $115(7.0)$ \\
\hline Asthma, N (\%) & $77(23.5)$ & $30(21.7)$ & $121(19.4)$ & $260(15.8)$ \\
\hline Fibromyalgia, N (\%) & $101(30.9)$ & $42(30.4)$ & $180(28.8)$ & $400(24.4)$ \\
\hline Diabetes, N (\%) & $91(27.8)$ & $40(29.0)$ & $162(26.0)$ & $363(22.1)$ \\
\hline Mood disorders, N (\%) & $58(17.7)$ & $37(26.8)$ & $135(21.6)$ & $326(19.9)$ \\
\hline Major depressive disorder, N (\%) & $51(15.6)$ & $35(25.4)$ & $118(18.9)$ & $278(16.9)$ \\
\hline No of previous biologics, N (\%) & & & & \\
\hline
\end{tabular}


Table 1 Continued

\begin{tabular}{cllll}
\hline Characteristics & IL-12/23 (N=327) & IL-17A* $(\mathbf{N}=138)$ & PDE4 (N=624) & TNF-alpha† (N=1641) \\
\hline 0 & $128(39.1)$ & $48(34.8)$ & $415(66.5)$ & $1183(72.1)$ \\
1 & $82(25.1)$ & $20(14.5)$ & $92(14.7)$ & $427(26.0)$ \\
$2+$ & $117(35.8)$ & $70(50.7)$ & $117(18.8)$ & $31(1.9)$ \\
\hline
\end{tabular}

*Secukinumab and ixekizumab.

†Infliximab, etanercept, adalimumab, certolizumab pegol and golimumab.

$\ddagger$ Suppressed due to small cell size $<11$, or to help mask other cells.

COPD, chronic obstructive pulmonary disease; DMARDS, disease-modifying antirheumatic drugs; ED, emergency department; IL, interleukin; NSAIDS, non-steroidal anti-inflammatory drugs; PDE4, phosphodiesterase-4; SD, standard deviation; TNF, tumour necrosis factor.

or IL-17A's (aRR $1.24,95 \%$ CI 0.86 to 1.79 ) compared with TNF- $\alpha$ 's. However, the comparative effectiveness of PDE4 treatment was lower than TNF- $\alpha$ (aRR $0.67,95 \%$ CI 0.46 to 0.96 ).

When the criterion of the effectiveness algorithm that disallowed any increase dose or frequency was relaxed, TNF- $\alpha$ outperformed IL-12/23 in the biologic-naïve cohort, while being comparable to IL-17A and PDE4. For the biologic-experienced cohort, effectiveness of TNF- $\alpha$ therapy was significantly higher compared with PDE4, significantly lower compared with IL-17A, and comparable to IL-12/23.

\section{DISCUSSION}

Despite the importance of biologic and disease-modifying drugs for the treatment of PsA, little is known regarding their real-world comparative effectiveness. In this analysis of a broad, diverse group of adults in the USA, we adapted a validated algorithm to assess the effectiveness of these exposures based on factors such as adherence, adding or switching biologic or PDE4, addition of new non-biologic DMARD, increase in dose or frequency and glucocorticoid use. We found that among biologic-naïve individuals, IL-12/23 inhibitors were less effective than TNF- $\alpha$ inhibitors, while IL-17A and PDE4 were comparable. Among biologic-experienced individuals, both IL-12/23 and PDE4 appeared less effective compared with TNF-, but only PDE4 was statistically significantly worse. Findings were robust and consistent in sensitivity analyses that allowed for relaxation of the dose and frequency escalation criterion with the possible exception that IL-17A therapy could be more effective than TNF- $\alpha$ treatment in certain patient subgroups. Although these outcomes are not validated for PsA, they share characteristics that would allow potential indirect interpretation of effectiveness.

Our results add to a growing body of literature, much of it based on randomised trials, quantifying the comparative effectiveness of different biologics and DMARDS for the treatment of inflammatory arthritis. For PsA,

Table 2 Unadjusted effectiveness of different treatments of interest among individuals with psoriatic arthritis $(\mathrm{N}=2730)^{*}$

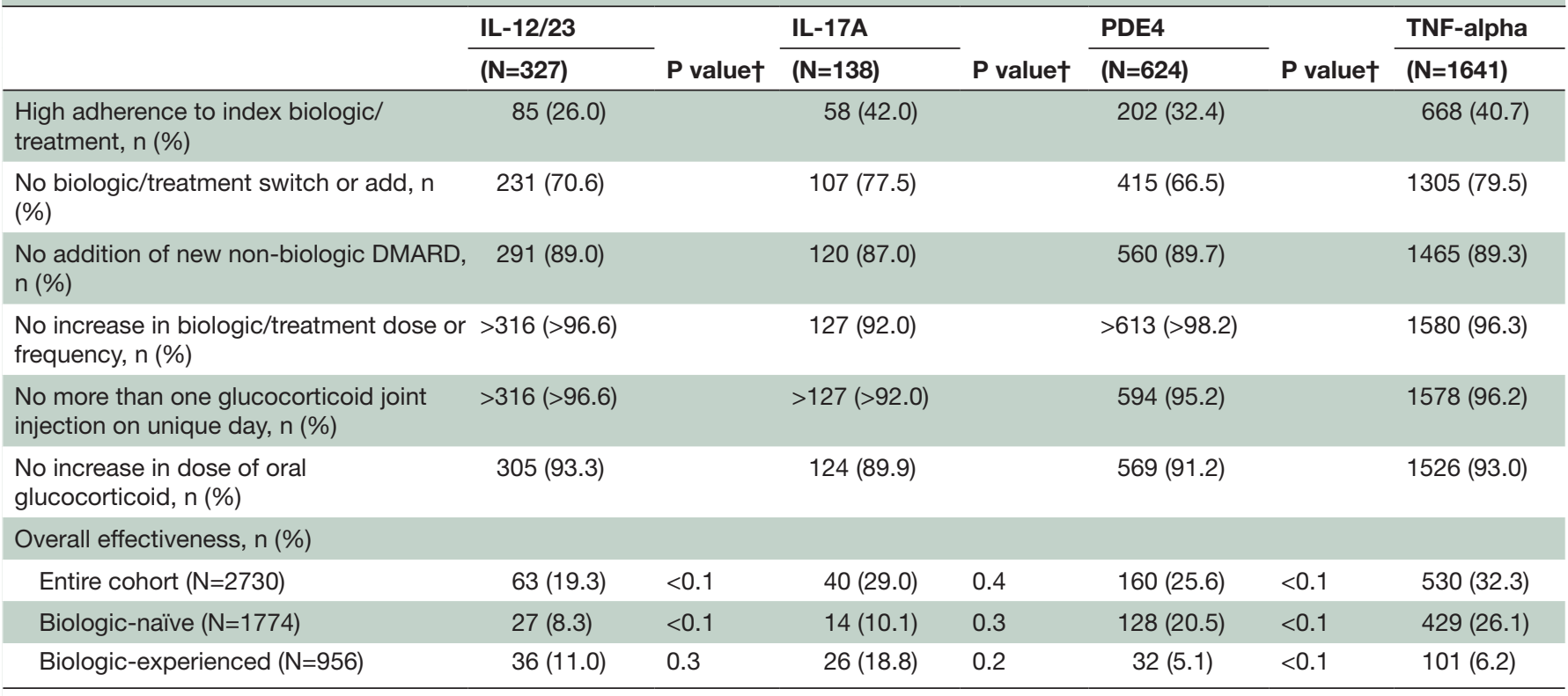

*Note criteria are not mutually exclusive.

†Pairwise $p$ value with TNF-alpha as reference.

DMARD, disease-modifying antirheumatic drugs; IL, interleukin; PDE4, phosphodiesterase-4; TNF, tumour necrosis factor. 
Table 3 Adjusted effectiveness comparison of full cohort between treatments of interest and tumour necrosis factor (TNF)-alpha inhibitors ( $\mathrm{N}=2730)$

\begin{tabular}{|c|c|c|c|c|}
\hline & \multicolumn{2}{|c|}{ Partially adjusted* } & \multicolumn{2}{|c|}{ Fully adjusted† } \\
\hline & $\mathbf{R R}$ & $95 \% \mathrm{Cl}$ & $\mathbf{R R}$ & $95 \% \mathrm{Cl}$ \\
\hline \multicolumn{5}{|l|}{ All criteria } \\
\hline TNF & Ref & & Ref & \\
\hline IL-12/23 & 0.59 & 0.47 to 0.75 & 0.75 & 0.59 to 0.95 \\
\hline IL-17A & 0.89 & 0.68 to 1.17 & 1.30 & 0.99 to 1.71 \\
\hline PDE4 & 0.79 & 0.68 to 0.92 & 0.90 & 0.77 to 1.04 \\
\hline
\end{tabular}

Sans criterion for dose or frequency escalation

\begin{tabular}{|lllll|} 
TNF & Ref & \multicolumn{3}{c}{ Ref } \\
\hline IL-12/23 & 0.57 & 0.46 to 0.72 & 0.72 & 0.57 to 0.91 \\
IL-17A & 1.04 & 0.83 to 1.32 & 1.50 & 1.18 to 1.91 \\
PDE4 & 0.75 & 0.65 to 0.87 & 0.85 & 0.73 to 0.99
\end{tabular}

*Adjusted for sex, age, race or ethnicity.

†Adjusted for sex, age, race or ethnicity, educational level, number of previous biologics, Deyo Charlson Comorbidity, sDMARD, oral glucocorticoid, NSAID, opioid, number of inpatient stays, number of ED stays, COPD, asthma, fibromyalgia, any diabetes, any mood disorder and major depressive disorder.

$\mathrm{Cl}$, confidence interval; COPD, chronic obstructive pulmonary disease; ED, emergency department; IL, interleukin; NSAID, nonsteroidal anti-inflammatory drugs; PDE4, phosphodiesterase-4; sDMARD, synthetic disease-modifying antirheumatic drugs.

previous studies that compared IL-12/13, IL-17A, PDE4 and $\mathrm{TNF}-\alpha$ treatments in various combinations have found that TNF- $\alpha$ 's generally performed similarly to IL-17A, while both groups outperform IL-12/23 and
PDE4..$^{78}$ 18-21 In a systematic review and network metaanalysis by Loos et al, ${ }^{22}$ IL-17A mostly performed better than ustekinumab, apremilast and some TNF- $\alpha$ inhibitors including etanercept and adalimumab on Psoriasis Area and Severity Index (PASI) 75 among adults with psoriasis. A randomised, open-label study comparing ixekizumab and adalimumab among biologic-naive patients found ixekizumab to be superior to adalimumab based on a composite outcome looking at both ACR50 and PASI100 at week $24 .{ }^{18}$ However, the composite result was based on noninferior ACR50 difference and a better PASI100 response for ixekizumab. ${ }^{18}$ The results held through week 52, where ixekizumab maintained superiority over adalimumab at achieving composite outcome. ${ }^{19} \mathrm{~A}$ separate trial comparing secukinumab against adalimumab among biologic-naïve patients found secukinumab was not significantly superior to adalimumab for ACR20 at 52 weeks, but hinted at higher proportion of secukinumab achieving the combined outcome of ACR50 and PASI $100 .{ }^{20}$ In an indirect meta-analysis study by Ungprasert et $a l,^{21}$ older TNF- $\alpha$ 's such as etanercept, infliximab, adalimumab and golimumab were comparable at attaining ACR20 response with secukinumab, while TNF- $\alpha$ 's and secukinumab generally performed better than ustekinumab, apremilast and certolizumab at certain doses. In a separate network meta-analysis by McInnes $e t a l,{ }^{7}$ they explored results based on previous biologic-experience and found that among biologicnaïve, infliximab and golimumab generally performed better than most treatments that were compared, but not secukinumab and adalimumab, based on ACR response rates. In the biologic-experienced population,

Table 4 Adjusted effectiveness comparison among biologic-naïve versus biologic-experienced individuals between treatments of interest and tumour necrosis factor (TNF)-alpha inhibitors

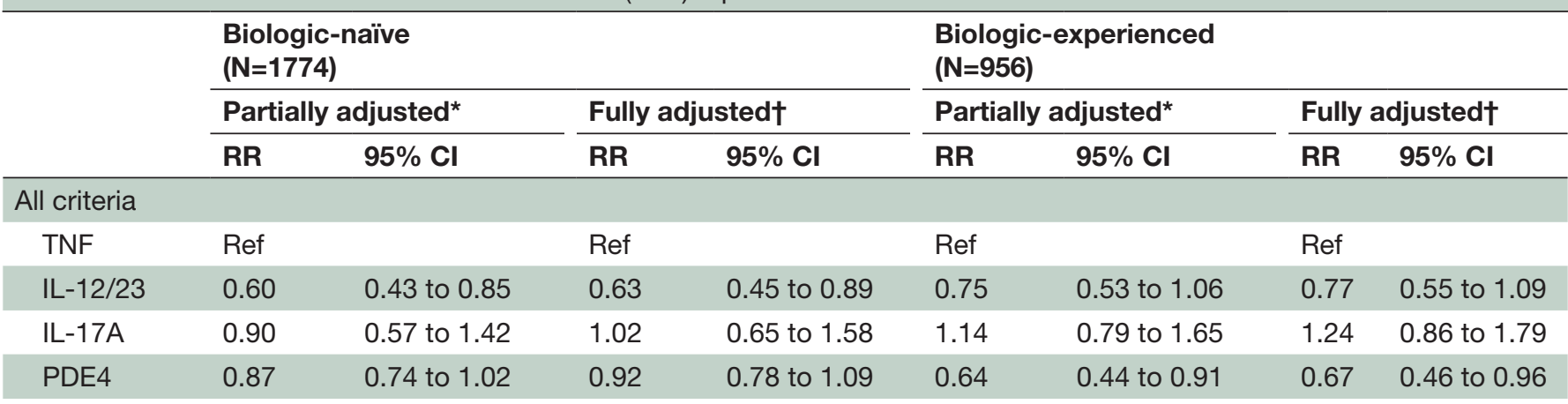

Sans criterion for dose or frequency escalation

\begin{tabular}{lllllllll}
\hline TNF & Ref & \multicolumn{3}{c}{ Ref } & \multicolumn{3}{c}{ Ref } \\
\hline IL-12/23 & 0.60 & 0.43 to 0.83 & 0.63 & 0.45 to 0.88 & 0.70 & 0.50 to 0.99 & 0.73 & 0.52 to 1.02 \\
\hline IL-17A & 1.03 & 0.69 to 1.54 & 1.17 & 0.80 to 1.72 & 1.33 & 0.96 to 1.84 & 1.44 & 1.03 to 1.99 \\
\hline PDE4 & 0.83 & 0.70 to 0.97 & 0.88 & 0.75 to 1.04 & 0.60 & 0.42 to 0.86 & 0.63 & 0.44 to 0.90 \\
\hline
\end{tabular}

*Adjusted for sex, age, race or ethnicity.

†Adjusted for sex, age, race or ethnicity, educational level, Deyo Charlson Comorbidity, sDMARD, oral glucocorticoid, NSAID, opioid, number of inpatient stays, number of ED stays, COPD, asthma, fibromyalgia, any diabetes, any mood disorder and major depressive disorder.

$\mathrm{CI}$, confidence interval; COPD, chronic obstructive pulmonary disease; ED, emergency department; IL, interleukin; NSAID, non-steroidal antiinflammatory drugs; PDE4, phosphodiesterase-4; sDMARD, synthetic disease-modifying antirheumatic drugs. 
certolizumab performed better than ustekinumab. ${ }^{7}$ Our results drew similar conclusions with respect to comparisons made between IL-12/23, IL-17A, PDE4 and TNF- $\alpha$ groups, with some additional insight towards differences in effectiveness based on prior biologic experience.

In terms of the generalisability of our results, findings from the biologic-experienced cohort may be most representative of patients who failed at least one TNF- $\alpha$ and who need alternative therapy for PsA. Further research exploring the comparative effectiveness of IL-12/23, IL-17A, PDE 4 and TNF- $\alpha$ set in large biologic-experienced cohorts could further explore which treatment may be more effective should an individual fail multiple lines of treatment, and account for which treatment that individual failed previously.

Our study has some limitations that must be considered. First, the effectiveness algorithm that we used excluded individuals with less than 1 year of follow-up. Only patients with continuous enrolment for at least 12 months after the index date were kept since they were assessed for treatment effectiveness based on a full 12-month period. Because of this, our analysis fails to represent the treatment experience among this subpopulation. Second, as with all observational studies, our findings are subject to omitted variable bias. For example, unmeasured factors, such as disease severity or concurrent medication use (eg, outpatient NSAIDs) not measured in this data, could confound the associations of interest. However, our estimates were similar in our partially adjusted and fully adjusted models, suggesting that there was not strong confounding, at least for factors that could be measured. Third, our analyses may be subject to misclassification for prior biologic exposure. For example, patients who we classified as biologic-naive and received IL-17A might actually be biologic-experienced, yet they received their prior biologic before enrolment in a health plan. This possibility likely would result in bias against the effectiveness of IL-17A therapy in the biologic-naïve subgroup, so results in the biologic-naïve strata should be considered a conservative estimate.

\section{CONCLUSIONS}

We assessed the comparative effectiveness of PsA therapy using a claims-based algorithm applied to large-scale health plan data. In this real-world data source, we demonstrated that our approach was able to mirror results from the few randomised controlled trials and meta-analyses that have been conducted. Among biologic-naive individuals, TNF- $\alpha$ inhibitors were found to outperform IL-12/23, and were comparable to IL-17A treatment and PDE4 at achieving effectiveness. For biologic-experienced individuals, TNF- $\alpha$ inhibitors were comparable to IL-17A, outperformed IL-12/23 and statistically significantly outperformed PDE4 therapy. Although our results indicate these biologics and PDE4 vary in effectiveness depending on prior biologic experience, head-to-head trials would help further validate findings and confirm true superiority in effectiveness between these treatments. Additional studies exploring comparative effectiveness between PsA treatments could help to inform treatment options and further identify PsA phenotypes more likely to respond to certain therapies. Finally, this methodological approach may be useful to health plans and large health systems to promote value-based care and reimbursement using administrative data as a proxy for clinical outcomes not routinely available.

\section{Author affiliations}

${ }^{1}$ Department of Epidemiology, Johns Hopkins University Bloomberg School of Public Health, Baltimore, Maryland, USA

${ }^{2}$ OptumLabs Visiting Fellow, OptumLabs, Eden Prairie, Minnesota, USA

${ }^{3}$ Center for Drug Safety and Effectiveness, Johns Hopkins Bloomberg School of Public Health, Baltimore, Maryland, USA

${ }^{4}$ Division of General Internal Medicine, Johns Hopkins Medicine, Baltimore, Maryland, USA

${ }^{5}$ Division of Clinical Immunology and Rheumatology, University of Alabama at Birmingham, Birmingham, Alabama, USA

${ }^{6}$ Department of Epidemiology, University of Alabama at Birmingham, Birmingham, Alabama, USA

Contributors JRC oversaw the project that provided the data for this paper. In addition, he participated in the methodological design and drafting of the manuscript. $\mathrm{HZ}$ led the development of the manuscript and participated in the analysis. JW conducted the analysis and drafted parts of the manuscript. GCA drafted parts of the paper and conducted the literature review. All authors participated in the response to reviewers.

Funding The authors have not declared a specific grant for this research from any funding agency in the public, commercial or not-for-profit sectors.

Competing interests GCA is past Chair of FDA's Peripheral and Central Nervous System Advisory Committee; has served as a paid advisor to IQVIA; is a consultant and holds equity in Monument Analytics, a health care consultancy whose clients include the life sciences industry as well as plaintiffs in opioid litigation; and is a member of OptumRx's National P\&T Committee. This arrangement has been reviewed and approved by Johns Hopkins University in accordance with its conflict of interest policies. JRC has received consultancies and funding for other studies from pharmaceutical companies that manufacture drugs of this class.

\section{Patient consent for publication Not required.}

Ethics approval Our analysis used deidentified data and was thus exempted from institutional review board review at the Johns Hopkins Bloomberg School of Public Health.

Provenance and peer review Not commissioned; externally peer reviewed.

Data availability statement All data relevant to the study are included in the article or uploaded as online supplemental information. This study includes deidentified data from OptumLabs.

Open access This is an open access article distributed in accordance with the Creative Commons Attribution Non Commercial (CC BY-NC 4.0) license, which permits others to distribute, remix, adapt, build upon this work non-commercially, and license their derivative works on different terms, provided the original work is properly cited, appropriate credit is given, any changes made indicated, and the use is non-commercial. See: http://creativecommons.org/licenses/by-nc/4.0/.

\section{ORCID iDs}

G Caleb Alexander http://orcid.org/0000-0002-5622-2986

Jeffrey R Curtis http://orcid.org/0000-0002-8907-8976

\section{REFERENCES}

1 European Medicines Agency. Stelara. European medicines Agency Available: https://www.ema.europa.eu/en/medicines/human/EPAR/ stelara [Accessed 19 Apr 2020].

2 European Medicines Agency. Cosentyx. European medicines Agency. Available: https://www.ema.europa.eu/en/medicines/ human/EPAR/cosentyx [Accessed 20 Apr 2020].

3 European Medicines Agency. Taltz. European medicines Agency. Available: https://www.ema.europa.eu/en/medicines/human/EPAR/ taltz [Accessed 20 Apr 2020]. 
4 Development history and FDA approval process for Stelara. Drugs. com. Available: https://www.drugs.com/history/stelara.html [Accessed 11 Nov 2018].

5 Development history and FDA approval process for Cosentyx. Drugs.com. Available: https://www.drugs.com/history/cosentyx.html [Accessed 11 Nov 2018].

6 Development history and FDA approval process for Taltz. Drugs. com. Available: https://www.drugs.com/history/taltz.html [Accessed 6 Nov 2019].

7 Mclnnes IB, Nash P, Ritchlin C, et al. Secukinumab for psoriatic arthritis: comparative effectiveness versus licensed biologics/apremilast: a network meta-analysis. J Comp Eff Res 2018;7:1107-23.

8 Kawalec P, Holko P, Moćko P, et al. Comparative effectiveness of abatacept, apremilast, secukinumab and ustekinumab treatment of psoriatic arthritis: a systematic review and network meta-analysis. Rheumatol Int 2018;38:189-201.

9 Kim SY, Solomon DH. Use of administrative claims data for comparative effectiveness research of rheumatoid arthritis treatments. Arthritis Res Ther 2011;13:129.

10 Curtis JR, Baddley JW, Yang S, et al. Derivation and preliminary validation of an administrative claims-based algorithm for the effectiveness of medications for rheumatoid arthritis. Arthritis Res Ther 2011;13:R155.

11 OptumLabs. OptumLabs and OptumLabs Data Warehouse (OLDW) descriptions and citation; 2020.

12 Wallace PJ, Shah ND, Dennen T, et al. Optum Labs: building a novel node in the learning health care system. Health Aff 2014;33:1187-94

13 Asgari MM, Wu JJ, Gelfand JM, et al. Validity of diagnostic codes and prevalence of psoriasis and psoriatic arthritis in a managed care population, 1996-2009. Pharmacoepidemiol Drug Saf 2013;22:842-9.

14 Walsh JA, Adejoro O, Chastek B, et al. Treatment patterns among patients with psoriatic arthritis treated with a biologic in the United
States: descriptive analyses from an administrative claims database. J Manag Care Spec Pharm 2018;24:623-31.

15 MAÁ M, Moura CS, Guerra SF. Effectiveness and safety of tofacitinib in rheumatoid arthritis: a cohort study. Arthritis Res Ther 2018;20.

16 Deyo RA, Cherkin DC, Ciol MA. Adapting a clinical comorbidity index for use with ICD-9-CM administrative databases. J Clin Epidemiol 1992;45:613-9.

17 Zou G. A modified poisson regression approach to prospective studies with binary data. Am J Epidemiol 2004;159:702-6.

18 Mease PJ, Smolen JS, Behrens F, et al. A head-to-head comparison of the efficacy and safety of ixekizumab and adalimumab in biological-naïve patients with active psoriatic arthritis: 24-week results of a randomised, open-label, blinded-assessor trial. Ann Rheum Dis 2020;79:123-31.

19 Smolen JS, Mease P, Tahir H, et al. Multicentre, randomised, openlabel, parallel-group study evaluating the efficacy and safety of ixekizumab versus adalimumab in patients with psoriatic arthritis naïve to biological disease-modifying antirheumatic drug: final results by week 52. Ann Rheum Dis 2020;79:1310-9.

20 Mclnnes IB, Behrens F, Mease PJ, et al. Secukinumab versus adalimumab for treatment of active psoriatic arthritis (exceed): a double-blind, parallel-group, randomised, active-controlled, phase 3B trial. Lancet 2020;395:1496-505.

21 Ungprasert P, Thongprayoon C, Davis JM. Indirect comparisons of the efficacy of biological agents in patients with psoriatic arthritis with an inadequate response to traditional disease-modifying antirheumatic drugs or to non-steroidal anti-inflammatory drugs: a metaanalysis. Semin Arthritis Rheum 2016;45:428-38.

22 Loos AM, Liu S, Segel C, et al. Comparative effectiveness of targeted immunomodulators for the treatment of moderate-to-severe plaque psoriasis: a systematic review and network meta-analysis. J Am Acad Dermatol 2018;79:135-44. 